\title{
Detecção de anticorpos Anti-HBV em pacientes atendidos por um laboratório privado em Parnaíba, Piauí
}

\author{
Detection of Anti-HBV antibodies in patients treated by a private laboratory in Parnaíba, Piauí \\ Detección de anticuerpos anti-VHB en pacientes atendidos por un laboratorio privado en Parnaíba,
} Piauí

\author{
Jadiel Silva Reis Filho \\ ORCID: https://orcid.org/0000-0002-4484-4876 \\ Universidade Federal do Piauí, Brasil \\ E-mail: jadielsilvareisfilho@hotmail.com \\ Raí Emanuel da Silva \\ ORCID: https://orcid.org/0000-0002-4266-0475 \\ Universidade Federal do Piauí, Brasil \\ E-mail: raiemanuel@ hotmail.com \\ Nathanael dos Santos Alves \\ ORCID: https://orcid.org/0000-0001-6945-6282 \\ Universidade Federal do Piaú, Brasil \\ E-mail: santosnathanael55@gmail.com \\ Cynthia Karolina Rodrigues do Nascimento \\ ORCID: https://orcid.org/0000-0002-2312-2364 \\ Universidade Federal do Piauí, Brasil \\ E-mail: cynthiakarolina7@gmail.com \\ Tássio Henrique Sousa Silva \\ ORCID: https://orcid.org/0000-0002-7792-2535 \\ Universidade Federal do Piauí, Brasil \\ E-mail: tassiohenriquee@outlook.com \\ Vaneska Magalhães Rios \\ ORCID: https://orcid.org/0000-0001-7922-8806 \\ Academia de Ciência e Tecnologia, Brasil \\ E-mail: vaneskarios@ hotmail.com \\ Ramila Beserra Marques \\ ORCID: https://orcid.org/0000-0002-6550-2971 \\ Centro Universitário UNINOVAFAPI, Brasil \\ E-mail: ramila-marques@hotmail.com. \\ Miguel Araújo Rios Neto \\ ORCID: https://orcid.org/0000-0002-7375-6445 \\ Academia de Ciência e Tecnologia, Brasil \\ E-mail: araujo-phb@hotmail.com \\ Fernanda Machado Fonseca \\ ORCID: https://orcid.org/0000-0002-5326-0776 \\ Universidade Federal do Triângulo Mineiro, Brasil \\ E-mail: fmachadofonseca@gmail.com
}

\begin{abstract}
Resumo
O objetivo do estudo foi avaliar a prevalência de marcadores sorológicos da infecção pelo vírus da hepatite B (HBV) em pacientes atendidos em um laboratório particular da cidade de Parnaíba, Piauí, no período de janeiro a dezembro de 2018. Trata-se de um estudo epidemiológico transversal, retrospectivo. Um total de 214 pacientes adultos foram incluídos. A presença do antígeno $\mathrm{HBsAg}$ foi detectada em quatro $(1,9 \%)$ pacientes enquanto que o marcador de imunidade anti-HBs foi detectado em 92 (43\%) pacientes. A presença do anti-HBc IgG foi detectada em $13(6,1 \%)$ casos. Em seis $(2,8 \%)$ pacientes foi possível detectar a presença concomitante do Anti-HBs e anti-HBc total. A idade dos pacientes variou de 2 a 86 anos (Média: 36,88 anos; DP: $\pm 14,77$ anos). A presença do HBsAg e do anti-HBc IgG que são marcadores da infecção pelo HBV foi detectada em maior frequência em pacientes com a faixa etária entre 46 a 59 anos. Por outro lado, a ausência desses marcadores foi observada em pacientes mais jovens. O presente estudo demonstrou uma prevalência considerada baixa para o $\mathrm{HBsAg}$ e anti-HBc, sugerindo que a população avaliada apresenta uma menor endemicidade da infecção pelo HBV.
\end{abstract}

Palavras-chave: Vírus da hepatite B; Epidemiologia; Estudos soroepidemiológicos. 


\begin{abstract}
The aim of the study was to evaluate the prevalence of serological markers of hepatitis B virus (HBV) infection in patients seen in a private laboratory in the city of Parnaíba, Piauí, from January to December 2018. This is an epidemiological cross-sectional and retrospective study. A total of 214 adult patients were included. The presence of the HBsAg antigen was detected in four (1.9\%) patients while the immunity marker anti-HBs was detected in 92 $(43 \%)$ patients. The presence of anti-HBc IgG was detected in $13(6.1 \%)$ cases. In six $(2.8 \%)$ patients it was possible to detect the concomitant presence of anti-HBs and total anti-HBc. The age of included patients ranged from 2 to 86 years (Mean: 36.88 years; SD: \pm 14.77 years). The presence of HBsAg and anti-HBc IgG, which are markers of HBV infection, was detected more frequently in patients aged 46 to 59 years. On the other hand, the absence of these markers was observed in younger patients. The present study demonstrated a low HBsAg and anti-HBc prevalence, suggesting that the population evaluated has a lower endemicity of HBV infection.
\end{abstract}

Keywords: Hepatitis B virus; Epidemiology; Seroepidemiologic studies.

\title{
Resumen
}

El objetivo del estudio fue evaluar la prevalencia de marcadores serológicos de infección por el virus de la hepatitis B (VHB) en pacientes atendidos en un laboratorio privado de la ciudad de Parnaíba, Piauí, de enero a diciembre de 2018. Se trata de un estudio epidemiológico transversal, retrospectivo. Se incluyeron un total de 214 pacientes adultos. Se detectó la presencia del antígeno $\mathrm{HBsAg}$ en cuatro $(1,9 \%)$ pacientes mientras que el marcador de inmunidad anti-HBs se detectó en 92 (43\%) pacientes. La presencia de IgG anti-HBc se detectó en 13 (6,1\%) casos. En seis $(2,8 \%)$ pacientes fue posible detectar la presencia concomitante de Anti-HBs y anti-HBc total. La edad de los pacientes osciló entre 2 y 86 años (Media: 36,88 años; DE: \pm 14,77 años). La presencia de HBsAg y anti-HBc IgG, que son marcadores de infección por VHB, se detectó con mayor frecuencia en pacientes de 46 a 59 años. Por otro lado, se observó la ausencia de estos marcadores en pacientes más jóvenes. El presente estudio demostró una prevalencia considerada baja para $\mathrm{HBsAg}$ y anti- $\mathrm{HBc}$, lo que sugiere que la población evaluada tiene menor endemicidad de infección por VHB.

Palabras clave: Virus de la hepatitis B; Epidemiología; Estudios seroepidemiológicos.

\section{Introdução}

As hepatites virais são doenças infecciosas provocadas por diferentes agentes etiológicos, que tem em comum o tropismo em infectar as células hepáticas. A distribuição da doença é universal e possui grande notabilidade pelo considerável número de pessoas atingidas assim como pela possibilidade de complicações das formas agudas e crônicas (Brasil, 2018; Novaes et al., 2021)

A hepatite B é causada pelo vírus B (HBV, do inglês hepatitis B virus), membro da família Hepadnaviridae, responsável por causar hepatite aguda e crônica em seres humanos. É frequentemente transmitido através de exposições percutâneas ou mucosas, pelo sangue ou outros fluidos corporais contaminados como sêmen, secreção vaginal e saliva. A transmissão pode ocorrer por meio de relações sexuais desprotegidas, pela transmissão vertical (mãe-filho), por contato próximo de pessoa a pessoa por cortes e feridas abertas, acidentes perfurocortantes, compartilhamento de agulhas, seringas ou qualquer outro instrumento destinado ao uso de drogas injetáveis (Brasil, 2018; Lopes \& Schinone, 2011; Kimberly \& Workowski, 2015).

Os marcadores sorológicos da infecção pelo vírus da hepatite B incluem antígenos virais (antígeno de superfície HBsAg e o antígeno E, HBeAg) e anticorpos como o anti-HBs (anticorpo contra o antígeno de superfície B), anti-HBc (anticorpo contra o antígeno do core viral) e anti-HBe (anticorpo contra o antígeno E). O HBsAg é o mais utilizado para rastrear a presença de infecção pelo HBV. A presença de HBeAg no soro de um hospedeiro é indicativo de replicação viral e está relacionada a maior taxa de infectividade. Assim, a detecção de todos os marcadores sorológicos é importante para o diagnóstico diferencial da doença e identificação de suas fases (Kimberly \& Workowski, 2015; Tian et al., 2019).

Apesar da disponibilidade de uma vacina eficaz, a hepatite B continua sendo um grave problema de saúde pública no mundo, pois afeta uma parcela significativa da população global. De acordo com a Organização Mundial de Saúde (OMS), estima-se que cerca de 257 milhões de pessoas vivem com a infecção crônica pelo HBV, o que corresponde a aproximadamente 3,5\% da população mundial. O relatório sobre hepatites da OMS evidencia que, em 2015, foram 
diagnosticadas apenas $9 \%$ de todas as infeções pelo HBV, e dentre as diagnosticadas, somente $8 \%$ das pessoas estavam sob a cobertura do tratamento (Yim \& Kim, 2019; WHO, 2017).

A relevância clínica das infecções causadas pelo HBV não se limita somente ao grande número de pessoas infectadas, mas também, às complicações das formas aguda e crônica da doença. $\mathrm{O}$ vírus causador da hepatite $\mathrm{B}$ é capaz de designar uma diversidade de apresentações clínicas que variam desde infecções inaparentes ou assintomáticas, até a evolução para casos mais críticos como o desenvolvimento de cirrose, insuficiência hepática e carcinoma hepatocelular (Burns \& Thompson, 2014; Brasil, 2018).

A prevalência da infecção crônica pelo HBV é inversamente correlacionada com a idade no momento da infecção. Assim, crianças nascidas de mães infectadas pelo vírus têm um risco de cronicidade de até 90\%, enquanto que apenas 5\% a 10\% das pessoas que adquirem a infecção na idade adulta evoluem para a cronicidade (Souza et al., 2012; Noordeen, 2015).

Segundo estudos epidemiológicos, o impacto do HBV no cenário mundial tem sido representado pelo alto índice de morbidade e mortalidade, sendo responsável por aproximadamente 780.000 óbitos anualmente. Embora os índices de mortalidade estejam aumentando, a taxa de incidência da doença está diminuindo devido a intensificação da cobertura vacinal contra o HBV entre crianças. Mundialmente, 84\% das crianças nascidas em 2015 tiveram acesso a vacinação contra a hepatite B. Desde o ano de introdução da vacina, 1981, até 2015, a proporção de crianças menores de cinco anos com novas infecções apresentou uma queda significativa, de 4,7\% para 1,3\% (WHO, 2017; Brasil, 2017; Araújo \& Silva, 2014).

No Brasil, de acordo com o boletim epidemiológico de hepatites virais do Ministério da Saúde, entre o período de 1999 a 2017 foram notificados mais de 218.000 casos de hepatite B. Do total de casos, a região Sudeste apresenta a maior taxa de prevalência da infecção, seguida pela região Sul. Desde 2011, os números de notificações tem permanecido estável, com poucas variações, atingindo uma média de 6,5 casos para cada 100 mil habitantes no país em 2017. Ainda conforme o boletim, a hepatite B é a segunda maior causa de óbitos entre as hepatites virais, representando $21,4 \%$ do total de casos registrados. No entanto, considerando a ocorrência de infecção assintomática em muitas pessoas e a notificação insuficiente de infecções sintomáticas, a frequência do HBV é provavelmente subestimada. Além disso, a maioria dos indivíduos desconhece sua condição sorológica, o que agrava ainda mais a transmissibilidade da doença (Brasil, 2018; Araújo et al., 2012; Melo et al., 2015; Brandão, Pfrimer, Martelli \& Turchi, 2015).

Diante do exposto, a investigação dos marcadores sorológicos para hepatite B é essencial, visto que o perfil sorológico dos indivíduos expostos pode determinar a história natural da doença e são indispensáveis para ampliar ações de vigilância epidemiológica e fortalecer estratégias de prevenção e controle. Assim, o presente estudo teve como objetivo avaliar a prevalência dos marcadores sorológicos para hepatite B em pacientes atendidos em um laboratório particular da cidade de Parnaíba, PI, no período de janeiro a dezembro de 2018.

\section{Metodologia}

Trata-se de um estudo epidemiológico transversal, retrospectivo, de abordagem quantitativa onde foram analisados resultados de testes sorológicos para o diagnóstico de hepatite B em pacientes atendidos em um laboratório particular da cidade de Parnaíba, Piauí. O laboratório que aceitou colaborar com o presente estudo assinou o Termo de Anuência autorizando a execução do estudo e o levantamento de dados.

Foram incluídos pacientes de ambos os sexos que compareceram ao laboratório para realizar exames sorológicos da hepatite B no período de janeiro a dezembro de 2018. A amostra foi representada por 214 pacientes. O Laboratório envolvido no estudo presta serviços particulares, para o sistema único de saúde - SUS, e possui convênio com várias empresas da região. 
Os exames incluídos na pesquisa foram, em sua grande maioria solicitados para realizar triagem de pacientes que faziam parte de algum grupo de risco para a infecção do HBV.

Foram excluídos pacientes que compareceram ao laboratório para a coleta de material fora do período de estudo e que por algum motivo a amostra não foi coletada e os exames não foram realizados.

O estudo foi desenvolvido em duas etapas: 1) Diagnóstico laboratorial: os pacientes compareceram previamente ao laboratório para a realização dos exames de sangue, onde foram utilizadas amostras de soro para deteç̧ão dos marcadores sorológicos do vírus da hepatite B (HBsAg, anti-HBs, anti-HBc IgM e anti-HBc IgG). Os testes foram realizados empregandose a metodologia de eletroquimioluminescência; 2) Coleta e análise dos dados cedidos pelo laboratório.

O laboratório disponibilizou quatro relatórios referente aos seguintes exames sorológicos: $\mathrm{HBsAg}$, anti-HBs, anti-HBc IgM e anti-HBc IgG. Nestes relatórios continham dados como data de nascimento do paciente, sexo, data da coleta e resultado do respectivo exame. A identidade dos pacientes foi preservada durante todo o período de estudo, não sendo permitido a obtenção de dados pessoais que permitissem a identificação dos mesmos.

Os dados foram analisados no software Statistical Package for the Social Sciences (SPSS), versão 23.0 pelo teste do qui-quadrado. Foram considerados significativos os valores de $\mathrm{P}<0,05$.

O presente estudo foi aprovado pelo Comitê de Ética em Pesquisa da Universidade Federal do Piauí (UFPI) (CAAE: 98443218.7.0000.5669). Vale ressaltar que a confidencialidade dos dados de identificação dos pacientes foi garantida durante todo o período de estudo, conforme disposto na Resolução nº. 196/96 do Conselho Nacional de Saúde.

\section{Resultados}

Foram incluídos 214 pacientes atendidos em um laboratório particular da cidade de Parnaíba e que realizaram exames de sangue para detecção de marcadores sorológicos para o vírus da hepatite B. A idade dos pacientes variou de 2 a 86 anos (média de 36,88 \pm 14,77 anos), 136 (63,6\%) pacientes eram do sexo feminino e 78 (36,4\%) eram do sexo masculino.

Os marcadores sorológicos para o HBV avaliados foram: HBsAg, anti-HBs, anti-HBc IgM e anti-HBc IgG. Em relação a prevalência sorológica do HBsAg, quatro (1,9\%) pacientes foram positivos e 200 (93,5\%) pacientes testaram negativos. Não foi possível determinar o status sorológico em um $(0,5 \%)$ paciente, sendo este classificado como indeterminado. Em nove $(4,2 \%)$ pacientes, não foi solicitado a pesquisa deste marcador sorológico. Dentre os pacientes positivos para HBsAg, um (25\%) tinha 42 anos, um (25\%) tinha 51 anos e dois (50\%) tinham 53 anos ( $\mathrm{P}=0,01)$.

A pesquisa do anti-HBc IgM foi realizada em 208 (97,2\%) pacientes e todos apresentaram sorologia negativa para este marcador. A distribuição de frequências absolutas e percentuais dos marcadores sorológicos dos pacientes avaliados está demonstrada na Tabela 1. 
Tabela 1. Prevalência dos marcadores sorológicos da hepatite B detectada nos pacientes avaliados $(\mathrm{N}=214)$.

Marcador Sorológico

\begin{tabular}{cccccc}
\cline { 2 - 5 } Resultado & HBsAg & Anti-HBs & Anti-HBc IgM & Anti-HBc IgG & Anti-HBs/Anti-HBc \\
& $\mathbf{N}(\%)$ & $\mathbf{N}(\%)$ & $\mathbf{N}(\%)$ & $\mathbf{N}(\%)$ & $\mathbf{N}(\%)$ \\
\hline Positivo & $4(1,9)$ & $92(43)$ & 0 & $13(6,1)$ & $6(2,8)$ \\
Negativo & $200(93,5)$ & $88(41,1)$ & $208(97,2)$ & $199(93)$ & $83(38,8)$ \\
Indeterminado & $1(0,5)$ & 0 & 0 & $2(0,9)$ & $2(0,9)$ \\
Não aplicável & $9(4,2)$ & $34(15,9)$ & $6(2,8)$ & 0 & $123(57,5)$ \\
\hline
\end{tabular}

Fonte: Dados da pesquisa (2021).

Quando avaliamos a positividade do anti-HBc IgG, dos 214 pacientes $13(6,1 \%)$ apresentaram sorologia positiva, 199 (93\%) foram negativos e em dois casos $(0,9 \%)$ não foi possível determinar o status sorológico. Dentre os pacientes com anti$\mathrm{HBc}$ IgG positivo, um (7,7\%) paciente tinha 42 anos, um (7,7\%) tinha 44 anos, sete $(53,8 \%)$ pacientes tinham idades entre 46 a 60 anos e quatro $(30,8 \%)$ pacientes tinham mais de 60 anos $(p=0.00)$. Quando avaliamos o anticorpo anti-HBs, $92(43 \%)$ pacientes tiveram reação positiva e em $88(41,1 \%)$ casos foi detectada reação negativa. Considerando os 214 pacientes incluídos no estudo, apenas seis $(2,8 \%)$ apresentaram positividade simultânea para os marcadores anti-HBc-total e anti-HBs (Tabela 1).

A faixa etária de 26 a 35 anos foi a que apresentou maior positividade (21\%; N=45) para o anti-HBs. No entanto, para o HBsAg e o anti-HBc IgG, a faixa etária com maior prevalência destes marcadores foi a de 46 a 59 anos com 1,4\% (3/214) e 3,3\% (7/214), respectivamente. O perfil sorológico para os marcadores da hepatite B foi avaliado de acordo com as faixas etárias e com o resultado dos testes (positivo, negativo, indeterminado e não aplicável - N/A) e está demonstrado na Tabela 2. 
Tabela 2. Perfil sorológico dos marcadores da hepatite B dos pacientes avaliados de acordo com as faixas etárias (N=214).

\begin{tabular}{|c|c|c|c|c|c|c|c|c|c|c|c|c|}
\hline \multirow{3}{*}{$\begin{array}{l}\text { Faixa etária } \\
\text { (anos) }\end{array}$} & \multicolumn{6}{|c|}{ Marcador Sorológico } & \multirow{2}{*}{\multicolumn{3}{|c|}{ "Anti-HBc IgG N (\%) }} & \multirow{2}{*}{\multicolumn{3}{|c|}{ "Anti-HBs N (\%) }} \\
\hline & \multicolumn{3}{|c|}{${ }^{*}$ HBsAg N $\quad$ (\%) } & \multirow[b]{2}{*}{ N/A } & \multicolumn{2}{|c|}{ Anti-HBc IgM N (\%) } & & & & & & \\
\hline & Positivo & Negativo & Indeterminado & & Negativo & N/A & $\overline{\text { Positivo }}$ & Negativo & Indeterminado & Positivo & Negativo & $\mathbf{N} / \mathbf{A}$ \\
\hline 0-17 & 0 & $6(2,8)$ & 0 & 0 & $6(2,8)$ & 0 & 0 & $6(2,8)$ & 0 & $2(0,9)$ & $3(1,4)$ & $1(0,5)$ \\
\hline $18-25$ & 0 & $29(13,6)$ & $1(0,5)$ & $1(0,5)$ & $30(14)$ & $1(0,5)$ & 0 & $31(14,5)$ & 0 & $16(7,5)$ & $10(4,7)$ & $5(2,3)$ \\
\hline $26-35$ & 0 & $79(36,9)$ & 0 & $7(3,3)$ & $84(39,3)$ & $2(0,9)$ & 0 & $84(39,3)$ & $2(0,9)$ & $45(21)$ & $28(13,1)$ & $13(6,1)$ \\
\hline $36-45$ & $1(0,5)$ & $46(21,5)$ & 0 & $1(0,5)$ & $46(21,5)$ & $2(0,9)$ & $2(0,9)$ & $46(21,5)$ & 0 & $16(7,5)$ & $29(13,6)$ & $3(1,4)$ \\
\hline 46-59 & $3(1,4)$ & $19(8,9)$ & 0 & 0 & $22(10,3)$ & 0 & $7(3,3)$ & $15(7)$ & 0 & $6(2,8)$ & $8(3,7)$ & $8(3,7)$ \\
\hline$\geq \mathbf{6 0}$ & 0 & $21(9,8)$ & 0 & 0 & $20(9,3)$ & $1(0,5)$ & $4(1,9)$ & $17(7,9)$ & 0 & $7(3,3)$ & $10(4,7)$ & $4(1,9)$ \\
\hline
\end{tabular}


Em relação ao perfil dos 214 pacientes incluídos no presente estudo, apenas 8 (3,7\%) eram de origem hospitalar, sendo que cinco eram provenientes de atendimento ambulatorial e três eram pacientes provenientes de atendimento na maternidade. Setenta e um (33,2\%) pacientes tiveram atendimento particular no laboratório e os demais (N=135; 63,1\%) possuíam algum convênio médico. Apenas $8(3,7 \%)$ pacientes tinham a suspeita de infecção como indicação para realização de sorologia para HBV, sendo todos eles oriundos de atendimento particular. Destes, um (12,5\%) paciente relatou ter caso da doença na família. O número de gestantes realizando a sorologia para HBV a partir de solicitação de exames pré-natal correspondeu a $18(8,4 \%)$. Os demais pacientes, compareceram por conta própria ao laboratório para realização dos exames. Em $146(68,2 \%)$ casos não foi possível obter maiores informações.

\section{Discussão}

O diagnóstico laboratorial da hepatite $\mathrm{B}$ é realizado por meio da detecção de anticorpos contra o vírus e antígenos virais, nas diferentes fases evolutivas da infecção por meio de técnicas sorológicas. Tais técnicas são fundamentais não apenas para a determinação do agente etiológico, mas também se mostram úteis no acompanhamento da infecção e na avaliação da condição clínica do paciente. Em casos suspeitos de infecção pelo vírus da hepatite B com amostras de sangue não reagentes à detecção do HBsAg, é recomendado a realização de testes moleculares para confirmação diagnóstica (Lopes \& Schinone, 2011; Brasil, 2018).

A detecção de anticorpos e antígenos do vírus B pode revelar diferentes estágios da infecção, como a fase aguda, crônica, uma resposta vacinal, ausência de contato prévio com o vírus, entre outras situações (Brasil, 2018). No presente estudo, foram analisadas a prevalência dos marcadores sorológicos HBsAg, anti-HBs, anti-HBc IgM e anti-HBc IgG ou total.

O HBsAg é o primeiro marcador a aparecer após a infecção pelo HBV, em torno de 30 a 45 dias, podendo permanecer detectável por até 120 dias. Sua persistência por mais de seis meses indica progressão da infecção crônica, e nos casos que evoluem para cura, deixa de ser detectado. Assim, a sua presença determina a condição de portador do HBV (sintomático ou assintomático), indicando a existência de risco de transmissão do vírus (Brasil, 2017; Moura \& Ferreira, 2019). A prevalência deste marcador detectada no presente estudo foi de $1,9 \%$, demonstrando que quatro pacientes estavam infectados no momento da coleta do material.

$\mathrm{O}$ anti-HBc IgG é um marcador que indica contato prévio com o HBV e permanece detectável por toda a vida no indivíduo que teve a infecção, representando memória imunológica mesmo naqueles casos de não cronicidade, ou seja, aqueles que eliminaram o vírus. Desta forma, o anti-HBc é amplamente utilizado para o desenvolvimento de estudos epidemiológicos. Por outro lado, o anti-HBc IgM é um marcador associado à presença do HBsAg e geralmente representa infecção aguda recente, sendo encontrado no soro até 32 semanas após a infecção. Sua permanência por um longo intervalo de tempo tem valor preditivo de progressão grave (Lopes \& Schinone, 2011; Kimberly \& Workowski, 2015; Brasil, 2018; Brasil, 2017). No presente estudo, não foi possível detectar a presença deste marcador sorológico, provavelmente devido a queda dos anticorpos IgM nos pacientes positivos para HBsAg e aumento na produção do IgG, como normalmente ocorre em resposta às infecções.

A presença de anti-HBs está associada à cura e ao desenvolvimento de imunidade à infecção por HBV. É o único marcador detectado em indivíduos que adquiriram imunidade através da vacinação e está presente em associação com anti$\mathrm{HBc}$ IgG naqueles que se recuperaram de uma infecção prévia por HBV. É detectado usualmente entre 1 a 10 semanas após o desaparecimento do HBsAg e sinaliza bom prognóstico. Geralmente, esses anticorpos são permanentes, contudo, podem se tornar indetectáveis anos após a resolução da infecção em pacientes imunossuprimidos (Kimberly \& Workowski, 2015; Brasil, 2017; Moura \& Ferreira, 2019). 
A detecção do anti-HBs encontrada no presente estudo indica que menos da metade da população avaliada possuía esses anticorpos no soro. Um fato que poderia explicar este achado pode estar relacionada com o protocolo de vacinação contra a hepatite B e que provavelmente não foi seguido corretamente pela população analisada. Desta forma, é possível que a amostragem estudada não tenha tido acesso às três doses da vacina ou não tenha aderido totalmente ao protocolo. Considerando a existência de uma vacina gratuita e eficaz na prevenção contra o HBV, era de se esperar uma maior prevalência deste marcador uma vez que o mesmo é marcador de imunidade. Adicionalmente, tem sido demonstrado que a completa adesão do protocolo de vacinação seguida da realização de exames laboratoriais permite a detecção de uma elevada prevalência do anti-HBs (Souza, Freitas, Araújo \& Gomes, 2015).

Os resultados do presente estudo demonstraram que a maioria da população avaliada era constituída por mulheres. Entretanto, outros estudos têm demonstrado uma maior prevalência da hepatite em B em homens (Araújo et al., 2020; Sato et al., 2020), o que pode estar relacionado com uma maior exposição desses indivíduos aos fatores de riscos. Contudo, ressalta-se que o padrão de utilização das instituições de saúde geralmente é composto em sua maioria por mulheres, que se fazem mais presentes e se preocupam com a própria saúde de modo mais frequente quando comparadas aos homens (Miranda; Passos; Figueiredo; Gaspar \& Yoshida, 2000; Levorato; Mello; Silva \& Nunes, 2014).

A endemicidade da infecção pelo HBV pode ser analisada de acordo com a prevalência do antígeno de superfície (HBsAg) na população ou com comprovação sorológica de infecção prévia (Aquino, Pegado, Barros \& Machado, 2008). A prevalência da infecção é considerada alta quando a porcentagem de HBsAg encontrada é maior que $7 \%$ ou quando a população apresenta taxa superior a $60 \%$ de infecção prévia (anti-HBc IgG positivo). Uma prevalência de infecção entre $20 \%$ e $60 \%$ para o anti-HBc IgG positivo e entre $2 \%$ a $7 \%$ para o $\mathrm{HBsAg}$, são consideradas regiões de endemicidade intermediária. As áreas classificadas como de baixa prevalência correspondem aquelas em que o HBsAg positivo é menor que 2\% (Ferreira \& Silveira, 2004).

No estado do Piauí, a prevalência da infecção pelo HBV por meio da detecção do HBsAg na população geral é de cerca de 2\% (Araújo et al., 2012). No presente estudo, o antígeno HBsAg foi detectado em 1,9\% dos casos. Esta informação assemelha-se com os achados de Barros, Ronchini e Soares (2018), que avaliaram gestantes atendidas na consulta pré-natal em um hospital universitário no Rio de Janeiro, onde a prevalência do HBsAg detectada também foi de 1,9\%. Por outro lado, no estudo de Oliveira et al. (2014) uma prevalência de 6\% foi detectada em 768 pacientes infectados pelo HIV, um achado comumente observado nesses casos. Um estudo realizado na cidade de Teresina (PI) avaliou 384 caminhoneiros que trafegam pela cidade. Foi demonstrado que o HBsAg foi positivo em 4,2\% dos casos (Rocha Filho, 2011). No estado do Tocantins, Villar et al. (2018) encontraram uma prevalência do HBsAg de 0,4\% em populações urbanas e ameríndias.

$\mathrm{O}$ anticorpo anti-HBc IgG foi detectado em 6,1\% na amostra estudada. Isso evidencia que, em algum momento da vida, estes pacientes tiveram contato com o vírus HBV, pois este marcador se encontra positivo em pacientes que estão na fase entre o desaparecimento dos antígenos e o aparecimento dos anticorpos de imunidade (Araujo \& Silva, 2014). Resultados semelhantes foram demonstrados em estudos realizados no Rio de Janeiro e entre gestantes assistidas nas maternidades públicas de São Luís, Maranhão (Pinto et al., 2015; Souza et al., 2012).

A prevalência de hepatites B na cidade de Buriticupu (MA), localizada na região leste da Amazônia brasileira, foi avaliada e uma taxa de positividade anti-HBc IgG de 40,7\% foi encontrada (El Khouri et al., 2010). Em contraste, Santos et al. (2012) encontraram uma frequência de anticorpos anti-HBc de 10\% em usuários do Sistema Único de Saúde atendidos no Laboratório Central do Piauí. É importante a detecção do anticorpo anti-HBc em trabalhos de determinação de prevalência, uma vez que este marcador permite conhecer o cenário da hepatite B na população estudada, e desta forma fornece uma visão 
mais real tanto da transmissão como também da circulação do vírus na comunidade (Passos et al., 1992; Livramento; Cordova; Spada \& Treitinger, 2011).

Um levantamento sorológico de base populacional realizado em indivíduos atendidos no Laboratório Central de Saúde Pública do Pará, verificou positividade de 3,9\% para o HBsAg, 37,7\% para o anti-HBc IgG e 3,1\% para o anti-HBc IgM (Aquino, Pegado, Barros \& Machado, 2008). Um perfil sorológico semelhante a este também foi observado por Martins et al. (2014) ao demonstrarem uma prevalência de 2,3\% para o HBsAg e 29,3\% para anti-HBc. Entretanto, no presente estudo, não foi detectada positividade para o anticorpo anti-HBc IgM, o que indica a inexistência de casos de infecção aguda por HBV na população avaliada no período do estudo.

$\mathrm{O}$ anti-HBs foi o marcador mais frequente na amostra avaliada, estando presente em $43 \%$ dos pacientes. A positividade desse marcador indica que esses pacientes apresentam imunidade contra o $\mathrm{HBV}$, sendo que esta pode ser atribuída a recuperação de uma infecção no passado ou ter sido adquirida após a imunização completa por meio do esquema de vacinação.

Em comparação a outros estudos, a prevalência do anti-HBs foi semelhante à encontrada por Pinto et al. (2015) em uma população universitária do Rio de Janeiro $(50,8 \%)$ e superior à verificada por Carvalho et al. (2017) em pessoas em situação de rua alojadas em um abrigo público da cidade de Goiânia, Goiás (19,5\%). Por outro lado, a imunidade adquirida naturalmente é estabelecida pela presença concomitante do anti-HBs e anti-HBc IgG (Araujo \& Silva, 2014). Neste estudo, foi observado que apenas 2,8\% dos pacientes apresentaram positividade simultânea para os marcadores anti-HBs e anti-HBc IgG, demonstrando que esses pacientes foram infectados pelo HBV, mas foram curados e naturalmente adquiriram imunidade ativa.

No Brasil, a prevalência dos marcadores de infecção do HBV é bastante heterogênea. Essas diferenças podem estar associadas a região e ao padrão de vida da população avaliada, assim como a um reflexo de endemicidade da infecção local e estilo de vida da área de estudo (Taye, Abdulkerim, \& Hussen, 2014).

A estratificação do perfil sorológico dos pacientes, por faixa etária, demonstra uma maior positividade de anticorpo anti-HBc IgG e antígeno HBsAg em indivíduos entre a faixa etária de 46 a 59 anos. Em contraste, esses marcadores estão ausentes em pacientes mais jovens (até 35 anos). $\mathrm{O}$ valor de 6,1\% para prevalência de anti-HBc $\operatorname{IgG}$ (contato prévio com HBV), bem como a presença de apenas quatro pacientes positivos para o HBsAg, todos acima de 40 anos de idade, evidenciam possivelmente o baixo nível de circulação do vírus B na população de estudo.

Portanto, a elevação da prevalência de acordo com a idade sugere o padrão de área de baixa circulação viral, em que a ausência de transmissão vertical ocasiona uma distribuição etária característica, com uma positividade que se apresenta escassa em idades precoces e tende a uma elevação lenta e gradativa conforme o aumento da faixa etária (Miranda et al., 2000). Nesse sentido, a transmissão sexual parece ter maior importância que a transmissão vertical na presente população de estudo, visto que os portadores do vírus HBV são potencialmente transmissores, em idade sexualmente ativa.

\section{Conclusão}

O presente estudo demonstrou uma prevalência considerada baixa para o HBsAg e anti-HBc sugerindo que a população avaliada apresenta uma menor endemicidade da infecção pelo HBV. Ainda que este estudo tenha sido conduzido apenas em um laboratório, as informações obtidas são úteis para a vigilância da situação do vírus da hepatite B. Um acompanhamento soro epidemiológico da infecção pelo vírus da hepatite $\mathrm{B}$, permite o estabelecimento de estratégias que visem auxiliar nos planos de vacinação e detectar áreas de maior endemicidade da doença, colaborando desta maneira com os órgãos de saúde local e regional. Além disso, a divulgação de dados epidemiológicos, como os apresentados no presente estudo, auxilia no conhecimento da circulação do vírus, a nível nacional. 
Dessa forma, a elaboração de estudos semelhantes, em períodos variados no futuro e com um maior número de pacientes avaliados, é necessária a fim de se determinar de forma representativa, a real circulação e transmissão desse agente na população.

\section{Referências}

Aquino, J. A., Pegado, K. S., Barros, L. P. \& Machado, L. F. A. (2008) Soroprevalência de infecções por vírus da hepatite B e vírus da hepatite C em indivíduos do Estado do Pará. Revista da Sociedade Brasileira de Medicina Tropical 41(4), 334-337. https://www.scielosp.org/article/rsp/2000.v34n3/286$291 / \mathrm{pt} /$.

Araújo, A. I. N., Oséas, J. M. F., Faria, J. C. B., Mendonça, B. P. N., Lima, C. M., Leite, F. P. P., Melo, L. A. (2020). Perfil epidemiológico das hepatites bec no estado do rio grande do norte. Revista Ciência Plural 6(3), 35-52. https://periodicos.ufrn.br/rcp/article/view/20537.

Araujo, T. M. E. \& Silva, N. C. (2014). Hepatite B: prevalência de marcadores sorológicos em profissionais de enfermagem de emergência. Revista Enfermagem UERJ 22(6), 834-839. https://pesquisa.bvsalud.org/portal/resource/pt/lil-749387.

Araújo, T. M. E., De Sá, L. C., Santos, A. S., Faustino, S. K. M., Tapety, F. I. \& Mesquita, G. V. (2012). Prevalence of Hepatitis B in users of Piauí Central Laboratory. Revista Enfermagem UERJ 20(20). 229-234.

Barros, M. M. O., Ronchini, K. R. O. M. \& Soares, R. L. S. (2018). Hepatitis B and C in Pregnant women attended by a prenatal program in an universitary Hospital in Rio de Janeiro, Brazil: Retrospective study of seroprevalence screening. Arquivos de Gastroenterologia 55(3), 267-273. https://www.scielo.br/pdf/ag/v55n3/1678-4219-ag-55-03-267.pdf.

Brandão, N. A. A., Pfrimer, I. A. H., Martelli, C. M. T. \& Turchi, M. D. (2015). Prevalence of hepatitis B and C infection and associated factors in people living with HIV in Midwestern Brazil. The Brazilian Journal of Infectious Diseases 19(4), 426-430. https://www.scielo.br/pdf/bjid/v19n4/1413-8670-bjid-1904-00426.pdf.

Brasil. (2017). Ministério da Saúde. Secretaria de Vigilância em Saúde. Coordenação-Geral da Epidemiologia em Serviços. Guia de Vigilância em Saúde: volume único [recurso eletrônico]. / Ministério da Saúde, Secretaria de Vigilância em Saúde, Coordenação-Geral da Epidemiologia em Serviços $2^{a}$ ed., 705. https://portalarquivos.saude.gov.br/images/pdf/2017/outubro/06/Volume-Unico-2017.pdf.

Brasil. (2017). Protocolo Clínico e Diretrizes Terapêuticas para Hepatite B e Coinfecções / Ministério da Saúde, Secretaria de Vigilância em Saúde, Departamento de DST, Aids e Hepatites $\quad$ Virais $\quad 1^{a}$ ed http://bvsms.saude.gov.br/bvs/publicacoes/protocolo_clinico_diretrizes_terapeuticas_hepatite_b_coinfeccoes.pdf.

Brasil. (2018). Boletim Epidemiológico da Hepatites Virais - 2018. Departamento de Vigilância, Prevenção e Controle das Infecções Sexualmente Transmissíveis, do HIV/Aids e das Hepatites Virais, Secretaria de Vigilância em Saúde, Ministério da Saúde 31(49), 1-72. http://www.aids.gov.br/ptbr/pub/2018/boletim-epidemiologico-de-hepatites-virais-2018.

Brasil. (2018). Manual Técnico para o Diagnóstico das Hepatites Virais / Ministério da Saúde, Secretaria de Vigilância em Saúde, Departamento de Vigilância, Prevenção e Controle das IST, do HIV/Aids e das Hepatites Virais $2^{a}$ ed., 123 . https://qualitr.paginas.ufsc.br/files/2018/08/manual_tecnico_hepatites_08_2018_web.pdf.

Burns, G. S. \& Thompson, A. J. (2014). Viral hepatitis B: clinical and epidemiological characteristics. Cold Spring Harbor Perspectives in Medicine 4(12), a024935-a024935. http://perspectivesinmedicine.cshlp.org/content/4/12/a024935.short.

Carvalho, P. M. R. S., Matos, M. A., Martins, R. M. B., Pinheiro, R. S., Caetano, K. A. A., Souza, M. M., et al. (2017). Prevalence, risk factors and hepatitis B immunization: helping fill the gap on hepatitis B epidemiology among homeless people, Goiânia, Central Brazil. Cadernos de Saúde Pública 33(7), e00109216. https://www.scielosp.org/article/csp/2017.v33n7/e00109216/.

El Khouri, M., Cordeiro, Q., Luz, D. A. B. P., Duarte, L. S., Gama, M. E. A. \& Corbett, C. E. P. (2010). Endemic hepatitis B and C virus infection in a Brazilian Eastern Amazon region. Arquivos de Gastroenterologia 47(1), 35-41. https://www.scielo.br/scielo.php?script=sci_arttext\&pid=S000428032010000100007.

Ferreira, C. T. \& Silveira, T. R. (2004). Hepatites virais: aspectos da epidemiologia e da prevenção. Revista Brasileira Epidemiologia 4 (7), $473-487$. https://www.scielo.br/pdf/rbepid/v7n4/10.pdf.

Kimberly, A. \& Workowski MD. (2015). Sexually Transmitted Diseases Treatment Guidelines, 2015. MMWR Recomm Rep 2015 64(RR 03), 1-137. https://www.cdc.gov/std/tg2015/tg-2015-print.pdf.

Levorato, C. D., Mello, L. M., Silva, A. S., Nunes, A. A. (2014). Fatores associados à procura por serviços de saúde numa perspectiva relacional de gênero. Ciência \& Saúde Coletiva 19, 1263-1274. https://www.scielosp.org/article/csc/2014.v19n4/1263-1274/pt/.

Livramento, A., Cordova, C. M. M., Spada, C. \& Treitinger, A. (2011). Seroprevalence of hepatitis B and C infection markers among children and adolescents in the southern Brazilian region. Revista do Instituto de Medicina Tropical de Sao Paulo 53(1), 13-17. https://www.scielo.br/scielo.php?pid=S0036$46652011000100003 \&$ script=sci_arttext.

Lopes, T. G. S. L. \& Schinone, M. I. (2011). Aspectos Gerais da Hepatite B. Revista Ciências Médicas e Biológicas 10(3), 337-344. https://cienciasmedicasbiologicas.ufba.br/index.php/cmbio/article/view/5899/4251. 
Martins, S., Livramento, A., Andrigueti, M., Kretzer, I. F., Machado, M. J., Spada, C. \& Aricio Treitinger (2014). The prevalence of hepatitis B virus infection markers and socio-demographic risk factors in HIV-infected patients in Southern Brazil. Revista da Sociedade Brasileira de Medicina Tropical 47(5), 552558. https://www.scielo.br/scielo.php?pid=S0037-86822014000500552\&script=sci_arttext.

Melo, L. V. L., Silva, M. A. B., Perdoná, G. S. C., Nascimento, M. M. P., Secaf, M., Monteiro, R. A., Martinelli, A. L. C. \& Passos, A. D. C. (2015). Epidemiological study of hepatitis B and C in a municipality with rural characteristics: Cássia dos Coqueiros, State of São Paulo, Brazil. Revista da Sociedade Brasileira Medicina Tropical 48(6). 674-681. https://www.scielo.br/scielo.php?pid=S0037-86822015000600674\&script=sci_arttext.

Miranda, L. V. G., Passos, A. D. C., Figueiredo, J. F. C., Gaspar, A. M. C. \& Yoshida, C. F. T. (2000). Marcadores sorológicos de hepatite B em indivíduos submetidos a exames de sangue em unidades de saúde. Revista de Saúde Pública 34(3), 286-291. https://www.scielosp.org/article/rsp/2000.v34n3/286$291 / \mathrm{pt} /$

Moura, J. P. \& Ferreira, A. S. A. S. (2019). Soroprevalência em testagem itinerante para sífilis, HIV e hepatites. Revista de Enfermagem UFPE On line (13), e239808. https://periodicos.ufpe.br/revistas/revistaenfermagem/article/view/239808/32497.

Noordeen F. (2015). Hepatitis B virus infection: An insight into infection outcomes and recent treatment options. VirusDisease 26(1-2), 1-8. https://link.springer.com/article/10.1007/s13337-015-0247-y.

Novaes, A. C., Tiroli, C. F., Ribeiro, B. Q., Ferreira, N. M. A., Furuya, R. K., Galhardi, L. C. F.. Pieri, F. M. (2021). Hepatites virais no contexto brasileiro: uma revisão integrativa. Research, Society and Development 10(1), e12510111579-e12510111579. https://rsdjournal.org/index.php/rsd/article/view/11579/10323.

Oliveira, E. H., Lima Verde, R. M. C., Pinheiro, L. M. L., Benghimil, M. G., Aragão, A. L. E. D., Lemos, J. A. R, Oliveira-Filho, A. B. \& Vallinoto, A. C. R. (2014). HBV infection in HIV-infected subjects in the state of Piauí, Northeast Brazil. Archives of Virology 159(5), 1193-1197. https://link.springer.com/article/10.1007\%2Fs00705-013-1921-2.

Passos, A. D. C., Gomes, U. A., Figueiredo, J. F. C., Nascimento, M. M. P., Oliveira, J. M., Gaspar, A. M. C. \& Yoshida, C. F. T. (1992). Prevalência de marcadores sorológicos de hepatite B numa pequena comunidade rural do Estado de São Paulo, Brasil. Revista de Saúde Pública 26(2), 119-124. https://www.scielosp.org/article/rsp/1992.v26n2/119-124/pt/.

Pinto, F. P. D., Ferreira Jr, O. C., Olmedo, D. B., Precioso, P. M., Barquette, F. R. S., Castilho, M. C., Silva, S. G. C. \& Pôrto, L. C. (2015). Prevalence of hepatitis B and C markers in a population of an urban university in Rio de Janeiro, Brazil: a cross-sectional study. Annals of Hepatology 14(6), 815-825. https://www.medigraphic.com/pdfs/hepato/ah-2015/ah156g.pdf.

Rocha Filho, D. R. (2011). Soroprevalência da infecção pelo vírus da hepatite B em caminhoneiros que trafegam por Teresina. (Dissertação de Mestrado). Universidade Federal do Piauí, Teresina, Piaú, $\quad$ Brasil. https://repositorio.ufpi.br/xmlui/bitstream/handle/12 3456789/1556/Disserta\%C3\%A7\%C3\%A3o.pdf?sequence=1

Sato, A. P. S., Koizumi, I. K., Farias, N. S. O., Silva, C. R. C., Cardoso, M. R. A., Figueiredo, G. M. (2020). Tendência de mortalidade por hepatites B e C no município de São Paulo, 2002-2016. Revista de Saúde Pública 54, 124. https://www.scielosp.org/article/rsp/2020.v54/124/pt/.

Souza, F. O., Freitas, P. S. P., Araújo, T. M. \& Gomes, M. R. (2015). Vacinação contra hepatite B e Anti-HBS entre trabalhadores da saúde. Cadernos Saúde Coletiva 23(2), 172-179. https://www.scielo.br/pdf/cadsc/v23n2/1414-462X-cadsc-23-2-172.pdf.

Souza, M. T., Pinho, T. L. R., Santos, M. D. C., Santos, A., Monteiro, V.L., Fonseca, L. M. B., Ferreira, P. A. M. \& Ferreira, A. S. P. (2012). Prevalence of hepatitis B among pregnant women assisted at the public maternity hospitals of São Luís, Maranhão, Brazil. The Brazilian Journal of Infectious Diseases 16(6), 517-520. https://reader.elsevier.com/reader/sd/pii/S1413867012002036?token=0C559FE2FA49AD204D70B8E81C5EEB474C0027F947A 93E3EB2A19B4DB45507A98C61D3582EA0498D942D15221262B1ED.

Taye, S., Abdulkerim, A. \& Hussen, M. (2014). Prevalence of hepatitis B and C virus infections among patients with chronic hepatitis at Bereka Medical Center, Southeast Ethiopia: a retrospective study. BMC Research Notes 7(1), 272. https://bmcresnotes.biomedcentral.com/articles/10.1186/1756-0500-7-272.

Tian, J., Xia, K., She, R., Li, W., Ding, Y., Wang, J., Chen, M. \& Yin, J. (2012). Detection of Hepatitis B virus in serum and liver of chickens. Virolology Journal 9(1), 2-4. https://virologyj.biomedcentral.com/articles/10.1186/1743-422X-9-2.

Villar, L. M., Milagres, F. A. P., Lampe, E., Cruz, H. M., Scaliono, L. P., Magalhães, M. A. F. M., Romão, A. R., Gracie, R. \& De Paula, V. S. (2018). Determination of hepatitis B, C and D prevalence among urban and Amerindian populations from the Eastern Brazilian Amazon: a cross sectional study. BMC Infectious Diseases 18(1), 411-420. https://link.springer.com/article/10.1186/s12879-018-3279-2.

World Health Organization. Global hepatitis report 2017. Geneva: WHO; 2017. https://apps.who.int/iris/bitstream/handle/10665/255016/9789241565455eng.pdf;jsessionid=F120ABB019FDB62CD95051A88C0F7B49?sequence $=1$.

Yim, S. Y. \& Kim, J. H. (2019). The epidemiology of hepatitis B virus infection in Korea. The Korean Journal of Internal Medicine 34(5), 945-953. https://www.ncbi.nlm.nih.gov/pmc/articles/PMC6718747/pdf/kjim-2019-007.pdf. 